\title{
ON THE RISK-ADJUSTED PRICING-METHODOLOGY-BASED VALUATION OF VANILLA OPTIONS AND EXPLANATION OF THE VOLATILITY SMILE
}

\author{
MARTIN JANDAČKA AND DANIEL ŠEVČOVIČ
}

Received 16 June 2004 and in revised form 28 January 2005

We analyse a model for pricing derivative securities in the presence of both transaction costs as well as the risk from a volatile portfolio. The model is based on the Black-Scholes parabolic PDE in which transaction costs are described following the Hoggard, Whalley, and Wilmott approach. The risk from a volatile portfolio is described by the variance of the synthesized portfolio. Transaction costs as well as the volatile portfolio risk depend on the time lag between two consecutive transactions. Minimizing their sum yields the optimal length of the hedge interval. In this model, prices of vanilla options can be computed from a solution to a fully nonlinear parabolic equation in which a diffusion coefficient representing volatility nonlinearly depends on the solution itself giving rise to explaining the volatility smile analytically. We derive a robust numerical scheme for solving the governing equation and perform extensive numerical testing of the model and compare the results to real option market data. Implied risk and volatility are introduced and computed for large option datasets. We discuss how they can be used in qualitative and quantitative analyses of option market data.

\section{Introduction}

In the past years, the Black-Scholes equation (see Black and Scholes [5]) and its generalizations for pricing derivatives have attracted a lot of attention from both theoretical as well as practical point of view. According to the classical Black-Scholes theory $[4,5,10,15,20,24]$, the present cost of an option is equal to the initial value of a solution to the so-called Black-Scholes equation. This theory is capable of valuing options and other derivative securities over moderate time intervals in which transaction costs and the risk from a volatile portfolio are negligible. On the other hand, if transaction costs like, for example, bid-ask spreads are taken into account, then the classical Black-Scholes theory is no longer applicable. In order to maintain the delta hedge, one has to make frequent portfolio adjustments yielding thus a substantial increase in transaction costs. On the other hand, rare portfolio adjustments leads to the increase of the risk from a volatile (unprotected) portfolio. 
One of the most important problems in the valuation of financial derivatives is a question how to incorporate both transaction costs and the risk arising from a volatile portfolio into the governing Black-Scholes equation. In [14], Kratka derived a mathematical model for pricing derivative securities in the case when both transaction costs as well as the risk from a volatile portfolio are taken into account. We modify Kratka's approach in order to derive a model which is mathematically well posed and scale invariant. These two important features were missing in the original model of Kratka. The model is based on the Black-Scholes parabolic PDE in which transaction costs are described by the Hoggard, Whalley, and Wilmott extension of the Leland model (cf. $[3,9,10,15,17])$ whereas the risk from a volatile portfolio is described by the average value of the variance of the synthesized portfolio. Transaction costs as well as the volatile portfolio risk depend on the time lag between two consecutive transactions. We define the total risk premium as the sum of transaction costs and the risk cost from the unprotected volatile portfolio. By minimizing the total risk premium functional, we obtain the optimal length of the hedge interval. It also gives us a new strategy for hedging derivative securities. These strategies are associated with a solution to a Cauchy problem for a fully nonlinear parabolic equation with a varying diffusion coefficient nonlinearly depending on the solution itself. The corresponding mathematical model will be henceforth referred to as the risk-adjusted pricing methodology model (RAPM). The resulting governing equation is scale invariant and can be mathematically treated. We present qualitative analysis of the governing equation in the case of a plain vanilla option (Call or Put). It can also be treated as the classical Black-Scholes equation with a non-constant diffusion coefficient, that is, volatility. It gives rise to explain analytically a striking phenomenon in option pricing theory-the volatility smile. Although we present the RAPM model for plain vanilla options only our approach can be extended to portfolios of Call and Put options by modifying the early exercise behavior and the so-called switching time (see Sections 2.5 and 2.6).

We furthermore derive a robust numerical scheme and we perform extensive numerical testing of the model. We compare the results to real option market datasets. We also introduce a concept of the so-called implied RAPM volatility and implied risk premium coefficient. Implied quantities are computed for large option datasets. We discuss how they can be used in qualitative analysis of option market datasets. The paper is organized as follows. In Section 2 we derive a scale-invariant risk-adjusted model for pricing plain vanilla options. We follow extended Leland's and modified Kratka's approaches in order to incorporate both transaction costs as well as the risk value arising from a volatile portfolio. Based on this model, it turns out that prices of options are solutions to a fully nonlinear parabolic partial differential equation. We discuss the choice of an optimal time interval between two consecutive portfolio adjustments. We also show scale invariance of the model. In Section 3 we analyze the resulting nonlinear partial differential equation. An important step is to transform a fully nonlinear parabolic equation for the option price into a quasilinear parabolic equation for the second derivative $\Gamma=\partial_{S}^{2} V$ of the option price $V$. We focus our attention on qualitative aspects of a solution like, for example, a priori bounds of a solution guaranteeing existence of a classical smooth solution in the case of a plain vanilla option. For such a quasilinear equation we can furthermore construct an effective numerical discretization scheme enabling us to find an 
approximate solution. A full space-time discretization scheme is discussed and analyzed in Section 4. Next, Section 5 contains results of numerical simulations and comparison of results based on the RAPM model to real market datasets. We also discuss how to calibrate the model. The implied RAPM volatility and implied risk premium are introduced. Finally, we present several numerical experiments comparing computational results to market quotes datasets.

\section{Derivation of a scale-invariant RAPM model}

Before describing the derivation of the RAPM model, we discuss the basic assumptions we will be making. Throughout the paper we assume that the asset price $S=S(t), t \geq 0$ follows a geometric Brownian motion with a drift $\rho$ and standard deviation $\sigma>0$, and it pays no dividends, that is,

$$
d S=\rho S d t+\sigma S d W
$$

where $d W$ denotes the differential of the standard Wiener process. This assumption is usually made when deriving the classical Black-Scholes equation (see, e.g., $[10,15])$. Notice that one of the greatest deficiencies of such an assumption is that the volatility $\sigma$ is constant. Moreover, it is not even possible to estimate this volatility in a reasonable way from historical data. Nevertheless, we will consider this assumption throughout the paper. The next step in the RAPM modeling should be therefore incorporation of a more realistic nonconstant volatility $\sigma$ into $(2.1)$ by means of, for example, time depending volatility $\sigma(t)$ or a volatility satisfying a stochastic differential equation of the mean reversion type (two or more factor models).

Similarly as in the derivation of the classical Black-Scholes equation, we construct a synthesized portfolio $\Pi$ consisting of one option with a price $V$ and $\delta$ assets with a price $S$ per one asset:

$$
\Pi=V+\delta S
$$

We recall that the key idea in the Black-Scholes theory is to examine the differential of (2.2). The right-hand side of (2.2) can be differentiated by using Itô's formula whereas the differential $\Delta \Pi(t)=\Pi(t+\Delta t)-\Pi(t)$ of the left-hand side can be expressed as follows:

$$
\Delta \Pi=r \Pi \Delta t
$$

where $r>0$ is a risk-free interest rate of a zero-coupon bond. In the real world, such a simplified assumption is not satisfied and a new term measuring the total risk should be added to (2.3). More precisely, the change of the portfolio $\Pi$ is composed of two parts: the risk-free interest rate part $r \Pi \Delta t$ and the total risk premium $r_{R} S \Delta t$, where $r_{R}$ is a risk premium per unit asset price. It means that $\Delta \Pi=r \Pi \Delta t+r_{R} S \Delta t$. The total risk premium $r_{R}$ consists of the transaction risk premium $r_{\mathrm{TC}}$ and the portfolio volatility risk premium $r_{\mathrm{VP}}$, that is, $r_{R}=r_{\mathrm{TC}}+r_{\mathrm{VP}}$. Hence

$$
\Delta \Pi=r \Pi \Delta t+\left(r_{\mathrm{TC}}+r_{\mathrm{VP}}\right) S \Delta t
$$


Our next goal is to show how these risk premium measures $r_{\mathrm{TC}}, r_{\mathrm{VP}}$ depend on other quantities, like, for example, $\sigma, S, V$, and derivatives of $V$. The problem can be decomposed in two parts: modeling transaction costs and modeling risk from a volatile portfolio.

2.1. Modeling transaction costs. In practice, we have to adjust our portfolio by frequent buying and selling of assets. In the presence of nontrivial transaction costs, continuous portfolio adjustments may lead to infinite total transaction costs. A natural way how to consider transaction costs within the frame of the Black-Scholes theory is to follow the well-known Leland approach [17] extended by Hoggard et al. (cf. [9, 15, 17]). In what follows, we recall crucial lines of the Hoggard, Whalley, and Wilmott derivation of Leland's model in order to show how to incorporate the effect of transaction costs into the governing equation. More precisely, we will derive the coefficient of transaction costs $r_{\mathrm{TC}}$ occurring in (2.4).

We denote by $C$ the round trip transaction cost per unit dollar of transaction. Then

$$
C=\frac{S_{\mathrm{ask}}-S_{\mathrm{bid}}}{S}
$$

where $S_{\text {ask }}$ and $S_{\text {bid }}$ are the so-called Ask and Bid prices of the asset, that is, the market price offers for selling and buying assets, respectively. Here $S=\left(S_{\text {ask }}+S_{\text {bid }}\right) / 2$ denotes the mid value. It means that the transaction cost is given by the value $C|k| S / 2$ where $k$ is the number of sold assets $(k<0)$ or bought assets $(k>0)$. The change of the portfolio $\Pi=$ $V+\delta S$ after a one-time step $\Delta t$ is $\Delta \Pi=\Delta V+\delta \Delta S-C|k| S / 2$. Clearly, the number $k$ of bought or sold assets depends on the one-time step change of $\delta$, that is, $k=\Delta \delta$. Therefore $\Delta \Pi=\Delta V+\delta \Delta S-C|\Delta \delta| S / 2$. We suppose that portfolio adjustments follow the so-called $\delta$-hedging strategy, that is, $\delta=-\partial_{S} V$. In the lowest order approximation in $\Delta t$, we obtain $\Delta \delta=-\sigma S \partial_{S}^{2} V \Delta W$. Since $W$ is the Wiener process, we have $E(|\Delta W|)=\sqrt{2 / \pi} \sqrt{\Delta t}$. If $\Delta t$ is small compared to $T-t$, Leland in [17] suggested to take the approximation $|\Delta W| \approx$ $E(|\Delta W|)$ (see also $[9$, page 25$]$ ) and thus

$$
\Delta \Pi=\Delta V+\delta \Delta S-r_{\mathrm{TC}} S \Delta t
$$

where the coefficient $r_{\mathrm{TC}}$ of transaction costs is given by the formula

$$
r_{\mathrm{TC}}=\frac{C \sigma S}{\sqrt{2 \pi}}\left|\partial_{S}^{2} V\right| \frac{1}{\sqrt{\Delta t}}
$$

(cf. [9, equation (3)]). Clearly, by increasing the time-lag $\Delta t$ between portfolio adjustments, we can decrease transaction costs. Therefore, in order to minimize transaction costs, we have to take a larger time lag $\Delta t$. On the other hand, as it will be shown in the next section, choosing a larger time lag $\Delta t$ could lead to a higher investor's exposure to the risk from an unprotected portfolio. 
2.2. Modeling risk from a volatile portfolio. In this section we focus our attention on the question how to include the risk from a volatile portfolio into the model. In the case when a portfolio consisting of options and assets is highly volatile, an investor usually asks for a price compensation.

Volatility of a fluctuating portfolio can be measured by the variance of relative increments of the replicating portfolio $\bar{\Pi}=V+\delta S$, that is, by the term $\operatorname{var}((\Delta \bar{\Pi}) / S)$. Hence it is reasonable to introduce the measure $r_{\mathrm{VP}}$ of the portfolio volatility risk as follows:

$$
r_{\mathrm{VP}}=R \frac{\operatorname{var}(\Delta \bar{\Pi} / S)}{\Delta t}
$$

In other words, $r_{\mathrm{VP}}$ is proportional to the variance of the relative change of a portfolio per time interval $\Delta t$. A constant $R$ is the so-called risk premium coefficient. It represents the marginal value of investor's exposure to a risk. Now applying Itô's formula to the differential $\Delta \bar{\Pi}=\Delta V+\delta \Delta S$, we obtain

$$
\Delta \bar{\Pi}=\left(\partial_{S} V+\delta\right) \sigma S \Delta W+\frac{1}{2} \sigma^{2} S^{2} \Gamma(\Delta W)^{2}+\mathscr{G},
$$

where $\Gamma=\partial_{S}^{2} V$ and $\mathscr{G}=\left(\partial_{S} V+\delta\right) \rho S \Delta t+\partial_{t} V \Delta t$ is a deterministic term, that is, $E(\mathscr{G})=\mathscr{G}$ in the lowest order $\Delta t$-term approximation. Thus

$$
\Delta \bar{\Pi}-E(\Delta \bar{\Pi})=\left(\partial_{S} V+\delta\right) \sigma S \phi \sqrt{\Delta t}+\frac{1}{2} \sigma^{2} S^{2}\left(\phi^{2}-1\right) \Gamma \Delta t
$$

where $\phi$ is a random variable with the standard normal distribution such that $\Delta W=$ $\phi \sqrt{\Delta t}$. Hence the variance of $\Delta \bar{\Pi}$ can be computed as follows:

$$
\begin{aligned}
\operatorname{var}(\Delta \bar{\Pi}) & =E\left[(\Delta \bar{\Pi}-E(\Delta \bar{\Pi}))^{2}\right] \\
& =E\left[\left(\left(\partial_{S} V+\delta\right) \sigma S \phi \sqrt{\Delta t}+\frac{1}{2} \sigma^{2} S^{2} \Gamma\left(\phi^{2}-1\right) \Delta t\right)^{2}\right] .
\end{aligned}
$$

Similarly, as in the derivation of the transaction costs measure $r_{\mathrm{TC}}$, we assume the $\delta$ hedging of portfolio adjustments, that is, we choose $\delta=-\partial_{S} V$. Since $E\left(\left(\phi^{2}-1\right)^{2}\right)=2$, we obtain an expression for the risk premium $r_{\mathrm{VP}}$ in the form

$$
r_{\mathrm{VP}}=\frac{1}{2} R \sigma^{4} S^{2} \Gamma^{2} \Delta t
$$

It means that the increase in the time lag $\Delta t$ between consecutive transactions leads to a linear increase of the risk from a volatile portfolio. In other words, larger time interval $\Delta t$ means higher risk exposure for an investor. 


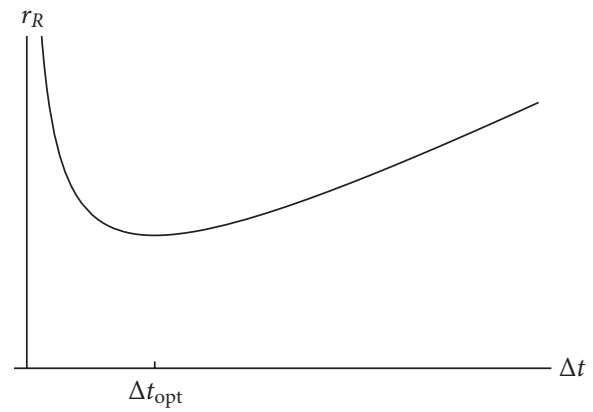

Figure 2.1. The total risk premium $r_{R}=r_{\mathrm{TC}}+r_{\mathrm{VP}}$ as a function of the time-lag $\Delta t$ between two consecutive portfolio adjustments.

2.3. Gamma hedging strategy based on the RAPM model. The total risk premium $r_{R}=$ $r_{\mathrm{TC}}+r_{\mathrm{VP}}$ consists of two parts: transaction costs premium $r_{\mathrm{TC}}$ and the risk from a volatile portfolio $r_{\mathrm{VP}}$ premium defined as in (2.7) and (2.12), respectively. We assume that an investor is risk aversive and wants to minimize the value of the total risk premium $r_{R}$. For this purpose one has to choose the optimal time-lag $\Delta t$ between two consecutive portfolio adjustments. As both $r_{\mathrm{TC}}$ as well as $r_{\mathrm{VP}}$ depend on the time lag $\Delta t$, so does the total risk premium $r_{R}$. In order to find the optimal value of $\Delta t$, we have to minimize the following function:

$$
\Delta t \longmapsto r_{R}=r_{\mathrm{TC}}+r_{\mathrm{VP}}=\frac{C|\Gamma| \sigma S}{\sqrt{2 \pi}} \frac{1}{\sqrt{\Delta t}}+\frac{1}{2} R \sigma^{4} S^{2} \Gamma^{2} \Delta t
$$

A graph of the function $\Delta t \mapsto r_{R}$ is depicted in Figure 2.1. The unique minimum of the function $\Delta t \mapsto r_{R}$ is attained at the time lag

$$
\Delta t_{\mathrm{opt}}=\frac{K^{2}}{\sigma^{2}|S \Gamma|^{2 / 3}}, \quad \text { where } K=\left(\frac{C}{R} \frac{1}{\sqrt{2 \pi}}\right)^{1 / 3} \text {. }
$$

For the minimal value of the function $\Delta t \mapsto r_{R}(\Delta t)$, we have

$$
r_{R}\left(\Delta t_{\mathrm{opt}}\right)=\frac{3}{2}\left(\frac{C^{2} R}{2 \pi}\right)^{1 / 3} \sigma^{2}|S \Gamma|^{4 / 3} .
$$

Remark 2.1. Since $S$ follows the geometric Brownian motion, in the lowest order approximation with respect to $\Delta t$, we have $E(|\Delta S| / S)=\sigma E(|\Delta W|)=\sqrt{(2 / \pi}) \sigma \sqrt{\Delta t}$. As a consequence from minimizing the total risk premium $r_{R}$, we can conclude that if $|\Delta S| / S \approx$ $K \sqrt{(2 / \pi})(S|\Gamma|)^{-1 / 3}$ (in the sense of expected values) then adjustment of the portfolio is needed. The portfolio is adjusted according to the $\delta$-hedging.

Remark 2.2. The approximation $|\Delta W| \approx E(|\Delta W|)=\sqrt{2 / \pi} \sqrt{\Delta t}$ used in Section 2.1 has been proposed by Leland and it holds for $0<\Delta t \ll 1$. For a larger $\Delta t$, one can however consider other approximations of $|\Delta W|$ like, for example, $|\Delta W| \approx \sqrt{E\left(|\Delta W|^{2}\right)}=\sqrt{\Delta t}$ which would lead to a different coefficient $r_{\mathrm{TC}}$ in (2.7). We consider an approximation 
of $|\Delta W|$ in the form $\phi(|\Delta W|) \approx E(\phi(|\Delta W|))$ where $\phi$ is a smooth increasing convex function, $\phi(0)=0$, that is, $|\Delta W| \approx \phi^{-1}(E(\phi(|\Delta W|)))$. If we insert such an approximation into the formula for $r_{\mathrm{TC}}$ (see (2.7)), we obtain $r_{\mathrm{TC}}=b \phi^{-1}(E(\phi(|\Delta W|))) / \Delta t$, where $b=C \sigma S|\Gamma| / 2$. We denote by $\widetilde{r}_{R}(\Delta t, \phi)$ the total risk premium in order to indicate that it depends on both the time lag $\Delta t$ as well as the way how we approximate $|\Delta W|$. Then $\tilde{r}_{R}(\Delta t, \phi)=a \Delta t+b \phi^{-1}(E(\phi(|\Delta W|))) / \Delta t$ where $a=R \sigma^{4} S^{2} \Gamma^{2} / 2$. By Jensen's inequality applied to a convex increasing function $\phi$, we have $E(\phi(|\Delta W|)) \geq \phi(E(|\Delta W|))$ and thus

$$
\tilde{r}_{R}(\Delta t, \phi) \geq a \Delta t+b \frac{E(|\Delta W|)}{\Delta t}=\tilde{r}_{R}(\Delta t, \mathrm{Id}) \equiv r_{R}(\Delta t)
$$

where Id is the identity function. Now, as $r_{R}(\Delta t) \geq r_{R}\left(\Delta t_{\text {opt }}\right)$, we have $\widetilde{r}_{R}\left(\Delta t_{\text {opt }}^{\phi}, \phi\right) \geq r_{R}\left(\Delta t_{\text {opt }}\right)$ where $\Delta t_{\mathrm{opt}}^{\phi}=\arg \min _{\Delta t>0} \tilde{r}_{R}(\Delta t, \phi)$. If we assume the approximation $|\Delta W| \approx E(|\Delta W|)=$ $\sqrt{2 / \pi} \sqrt{\Delta t}$ for all $\Delta t$ and we take the optimal time lag $\Delta t_{\text {opt }}$ as in (2.14), then we can achieve the lowest possible total risk premium among all convex increasing approximations $\phi$ of the stochastic term $|\Delta W|$. As we are minimizing the total risk premium in the RAPM model, the previous argument can justify such an approximation of $|\Delta W|$ made in Section 2.1.

2.4. Risk-adjusted Black-Scholes equation. Taking into account both transaction costs as well as risk from a volatile portfolio effects, we have shown that the equation for the change $\Delta \Pi$ of a portfolio $\Pi$ read as

$$
\Delta \Pi=\Delta V+\delta \Delta S-r_{R} S \Delta t
$$

where $r_{R}$ represents the total risk premium, $r_{R}=r_{\mathrm{TC}}+r_{\mathrm{VP}}$. On the other hand, by the no-arbitrage principle, the change $\Delta \Pi$ in the portfolio $\Delta \Pi$ equals the change $r \Pi \Delta t$ of secure bonds with the interest rate $r>0$. Applying Itô's lemma to the smooth function $V=V(S, t)$ and assuming the $\delta$-hedging strategy for the portfolio adjustments, we finally obtain the following generalization of the Black-Scholes equation for valuing options:

$$
\partial_{t} V+\frac{\sigma^{2}}{2} S^{2} \partial_{S}^{2} V-r_{R} S=r\left(V-S \partial_{S} V\right)
$$

By taking the optimal value of the total risk coefficient $r_{R}$ derived as in (2.15), the option price $V$ is a solution to the following nonlinear parabolic equation.

Risk-adjusted Black-Scholes equation.

$$
\partial_{t} V+\frac{\sigma^{2}}{2} S^{2}\left(1-\mu(S \Gamma)^{1 / 3}\right) \Gamma=r\left(V-S \partial_{S} V\right)
$$

where

$$
\Gamma=\partial_{S}^{2} V, \quad \mu=3\left(\frac{C^{2} R}{2 \pi}\right)^{1 / 3} .
$$

Here and after we will denote by $x^{1 / 3}$ the signed power function, that is, $x^{p}=|x|^{p-1} x=$ $|x|^{p} \operatorname{sign}(x)$ for all $x \in R, p>0$. In the case where there are neither transaction costs 


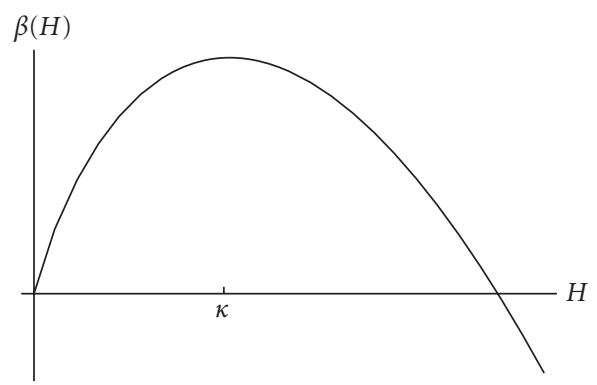

Figure 2.2. The function $\beta(H)=\left(\sigma^{2} / 2\right)\left(1-\mu H^{1 / 3}\right) H$. Equation (2.19) is parabolic for $H=S \Gamma<\kappa=$ $(3 /(4 \mu))^{3}$.

$(C=0)$ nor the risk from a volatile portfolio $(R=0)$, we have $\mu=0$. Then (2.19) reduces to the original Black-Scholes linear parabolic equation

$$
\partial_{t} V+\frac{\sigma^{2}}{2} S^{2} \Gamma=r\left(V-S \partial_{S} V\right)
$$

We note that (2.19) is a backward parabolic PDE if and only if the function

$$
\beta(H)=\frac{\sigma^{2}}{2}\left(1-\mu H^{1 / 3}\right) H
$$

(see Figure 2.2) is an increasing function in the variable $H:=S \Gamma=S \partial_{S}^{2} V$. Hence, in order to verify parabolicity of (2.19), we have to assume the following condition:

$$
S \Gamma<\kappa:=\left(\frac{3}{4 \mu}\right)^{3} .
$$

We remind ourselves that the terminal payoff for a Call option at $t=T$ is given by $V(S, T)=\max (S-E, 0)$. For a Put option one has $V(S, T)=\max (E-S, 0)$. Here and after, $E$ denotes the exercise price and $T$ stands for the exercise time. Furthermore, a Call option price $V(S, t)$ is subject to boundary conditions $V(0, t)=0, V(S, t) / S \rightarrow 1$ as $S \rightarrow \infty$, $t \in(0, T)$. Similarly, the Put option price satisfies $V(0, t)=E e^{-r(T-t)}, V(S, t) \rightarrow 0$ as $S \rightarrow \infty$ (cf. $[10,15])$.

If we consider prices of either Call or Put options on assets paying no dividends satisfying the classical Black-Scholes equation (2.21), then the term $S \Gamma=S \partial_{S}^{2} V(S, t)$ becomes infinite at $S=E$ for $t \rightarrow T^{-}$and the above condition is violated. This is why we have to examine the early exercise behavior of a solution in a more detail.

2.5. Early exercise behavior. Our next goal is to analyze the behavior of the option price $V=V(S, t)$ near the exercise time $T$, that is, when $T-t$ is small. Recall that we have applied Leland's methodology in modeling transaction costs. In this approach one has to assume that the time-lag $\Delta t$ between consecutive portfolio adjustments is small compared to $T-t$ (see $[10,15,17])$. A natural way to satisfy the condition $\Delta t_{\text {opt }} \ll T-t$ is to disallow portfolio adjustments when the time $t$ is close to the exercise time $T$. The idea 
is to divide the time interval $(0, T)$ into two parts: $(1)$ the interval $\left(0, t_{*}\right)$ where the riskadjusted Black-Scholes equation takes place; (2) the interval $\left(t_{*}, T\right)$ where no portfolio adjustments are allowed. Here and after we denote by $t_{*}$ the so-called switching time to be determined later. It should be close to the exercise time $T$. Within the time interval $\left(t_{*}, T\right)$, we assume that the asset price $S_{t}, t \in\left(t_{*}, T\right)$, follows the geometric Brownian motion of the form (2.1). Since $t_{*} \approx T$, the drift $\varrho$ is assumed to be known and it coincides with the risk-free zero coupon bond rate $r$, that is, $d S=r S d t+\sigma S d w$. Since the portfolio adjustments are disallowed within the interval $\left(t_{*}, T\right)$, it is natural to assume that the option price $V\left(S, t_{*}\right)$ at the time $t_{*}$ is simply an expected value of $V\left(S_{T}, T\right)$ subject to the condition $S_{t_{*}}=S$ discounted by the risk-free interest rate $r$, that is,

$$
V\left(S, t_{*}\right)=e^{-r\left(T-t_{*}\right)} E\left(V\left(S_{T}, T\right) \mid S_{t_{*}}=S\right) .
$$

Since $S_{t}$ follows the geometric Brownian motion (2.1) with $\varrho=r$ and $S_{t_{*}}=S$, it follows from Itô's formula that $S_{t}=S e^{X_{t}}$ where $d X=\left(r-\sigma^{2} / 2\right) d t+\sigma d w$ and $X_{t_{*}}=0$. Hence the cummulative distribution function $F_{T}(s)=P\left(S_{T}<s\right)$ is given by

$$
F_{T}(s)=\frac{1}{\sqrt{2 \pi\left(T-t_{*}\right)}} \int_{-\infty}^{\left(\ln (s / S)-\left(r-\sigma^{2} / 2\right)\left(T-t_{*}\right)\right) / \sigma} e^{-\xi^{2} / 2\left(T-t_{*}\right)} d \xi .
$$

In the case of a Call option, we have $V\left(S_{T}, T\right)=\max \left(S_{T}-E, 0\right)$. After some calculations we obtain

$$
E\left(V\left(S_{T}, T\right) \mid S_{t_{*}}=S\right)=\int_{-\infty}^{\infty} \max (s-E, 0) F_{T}^{\prime}(s) d s=S e^{r\left(T-t_{*}\right)} N\left(d_{1}\right)-E N\left(d_{2}\right),
$$

where $d_{1}, d_{2}$ are defined as follows:

$$
d_{1}=\frac{\ln (S / E)+\left(r+\sigma^{2} / 2\right)\left(T-t_{*}\right)}{\sigma \sqrt{T-t_{*}}}, \quad d_{2}=d_{1}-\sigma \sqrt{T-t_{*}},
$$

and $N^{\prime}(d)=(1 / \sqrt{2 \pi}) e^{-d^{2} / 2}$ is the density function of the standard normal distribution. Hence

$$
V\left(S, t_{*}\right)=S N\left(d_{1}\right)-E e^{-r\left(T-t_{*}\right)} N\left(d_{2}\right) .
$$

Notice that expression (2.28) is nothing else but the valuation formula for pricing a European Call option obtained from a solution to the classical Black-Scholes equation (2.21). Furthermore, we have $\partial_{S} V\left(S, t_{*}\right)=N\left(d_{1}\right)$ and

$$
S \Gamma\left(S, t_{*}\right)=\frac{N^{\prime}\left(d_{1}\right)}{\sigma \sqrt{T-t_{*}}}, \quad \max _{S>0} S \Gamma\left(S, t_{*}\right)=\frac{1}{\sqrt{2 \pi \sigma^{2}\left(T-t_{*}\right)}} .
$$

Exactly the same expression for $S \Gamma\left(S, t_{*}\right)$ is true for a Put option. It it worth to emphasize that the maximal value $\max _{S>0} S \Gamma\left(S, t_{*}\right)$ does not depend on the interest rate $r$. Hence, if we take an arbitrary interest rate $\varrho$ in the stochastic equation $d S=\varrho S d t+\sigma S d W$, then the maximum value $\max _{S>0} S \Gamma\left(S, t_{*}\right)$ depends on the volatility $\sigma$ and the time to expiry $T-t_{*}$. 
2.6. Switching time. It remains to determine the switching time $t_{*}$. It divides the interval $(0, T)$ into two subintervals: $\left(0, t_{*}\right)$ and $\left(t_{*}, T\right)$. The idea is rather simple and consists in finding the very last portfolio adjustment moment $0<t_{*}<T$ before the expiry $T$. It can be done by assuming our hedging strategy follows the optimal time lag stepping $\Delta t_{\mathrm{opt}}$ derived as in (2.14). More precisely, the switching time $t_{*}$ can be determined from the implicit equation

$$
T-t_{*}=\min _{S>0} \Delta t_{\mathrm{opt}}\left(S, t_{*}\right) .
$$

Combining (2.14) and (2.29), we obtain

$$
\begin{aligned}
T-t_{*} & =\min _{S>0} \Delta t_{\mathrm{opt}}\left(S, t_{*}\right) \\
& =K^{2} \sigma^{-2}\left(\max _{S>0} S \Gamma\left(S, t_{*}\right)\right)^{-2 / 3} \\
& =K^{2} \sigma^{-2}\left(2 \pi \sigma^{2}\left(T-t_{*}\right)\right)^{1 / 3} .
\end{aligned}
$$

Taking into account expression (2.14) for the constant $K$, we can determine the switching time $t_{*}$ from the equation

$$
T-t_{*}=\frac{C}{R \sigma^{2}} .
$$

As $t_{*}$ must be positive, we have $T-t_{*}<T$. Hence we have to require the following condition:

$$
C<\sigma^{2} R T \text {. }
$$

This way we have determined the switching time $t_{*}$ and the time interval $\left(0, t_{*}\right)$ on which the option price $V(S, t)$ satisfies the risk-adjusted Black-Scholes equation (2.19) subject to the terminal condition $V\left(S, t_{*}\right)$ (see (2.28)). In order to guarantee the existence of a solution to (2.19), we have to verify condition (2.23) ensuring its backward parabolicity. A maximum principle argument (cf. [19]) applied to an equation for the new variable $H=$ $S \Gamma$ derived in Section 3.2 enables us to conclude that $(2.23)$ is satisfied for $H=S \Gamma(S, t)$, $S>0,0<t<t_{*}$, if and only if (2.23) is fulfilled at the terminal time $t_{*}$, that is,

$$
\max _{S>0} S \Gamma\left(S, t_{*}\right)<\kappa:=\left(\frac{3}{4 \mu}\right)^{3} .
$$

With regard to (2.32), (2.29), and expression (2.20), we can conclude that the riskadjusted Black-Scholes equation (2.19) is backward parabolic provided that

$$
C R<\frac{\pi}{8} .
$$

Throughout the rest of the paper, we will assume that condition (2.35) for the product of the risk measure $R$ and transaction cost measure $C$ as well as condition (2.33) are satisfied.

Now we are in a position to introduce a notion of a solution to the risk-adjusted BlackScholes equation. 
Definition 2.3. By a solution to the risk-adjusted Black-Scholes equation we mean a continuous function $V=V(S, t), S \in(0, \infty), t \in[0, T]$, satisfying boundary conditions, the terminal payoff condition at $t=T$, and such that

(a) $V(S, t)$ is a classical (smooth) solution to the Black-Scholes equation

$$
\partial_{t} V+\frac{\sigma^{2}}{2} S^{2} \Gamma=r\left(V-S \partial_{S} V\right), \quad S>0
$$

on the time interval $\left(t_{*}, T\right)$ and it satisfies the prescribed terminal payoff condition at $t=T$,

(b) $V(S, t)$ is a classical (smooth) solution to the equation

$$
\partial_{t} V+S \beta(S \Gamma)=r\left(V-S \partial_{S} V\right), \quad S>0,
$$

on the time interval $t \in\left(0, t_{*}\right)$ satisfying $V\left(S, t_{*}\right)=V_{*}(S)$, where $t_{*}=T-C /\left(R \sigma^{2}\right)$ is a switching time and $V_{*}(S)=\lim _{t \rightarrow t_{*}^{+}} V(S, t)$.

Remark 2.4. Leland in [17] claimed that in the presence of transaction costs a Call option can be perfectly hedged using the Black-Scholes delta hedging with a modified volatility. Kabanov and Safarian [12] have shown failure of Leland's statement and they proved that the limiting hedging error in Leland's strategy is equal to zero only in the case where the level of transaction costs tends to zero (sufficiently fast) in the limit when the time lag between two consecutive portfolio adjustments goes to zero. On the other hand, they have shown that the plain vanilla option is always underpriced (i.e., the hedging error is negative) in such a limit (see also Grandits and Schachinger [8]). In the RAPM model, we do not use the wrong statement made by Leland. We only use Leland's approximation $|\Delta W| \approx E(|\Delta W|)=\sqrt{2 / \pi} \sqrt{\Delta t}$ which can be justified, at least partially, by Remark 2.2. Moreover, in the RAPM model we are not involved with the limiting case when the time lag goes to zero because $\Delta t_{\mathrm{opt}}$ is always bounded from below by a positive constant $C /\left(R \sigma^{2}\right)$. It follows from (2.30) and (2.35).

2.7. Scale-invariance property. The governing equation (2.19) has a natural scale invariance property. Indeed, we multiply the asset and option prices by the same scaling factor $\kappa>0$. Denote $\tilde{S}=\kappa S, \tilde{V}=\kappa V$. Then $\tilde{S} \widetilde{\Gamma}=\tilde{S} \partial_{\widetilde{S}}^{2} \widetilde{V}=S \partial_{S}^{2} V=S \Gamma$, that is, the term $S \Gamma$ remains unchanged after scaling of $S$ and $V$ by a factor $\kappa>0$. Therefore the scaled option price $\tilde{V}$ satisfies the same governing equation (2.19) in which we change the variable $S$ to $\widetilde{S}$. This is a very important property of the governing equation which was missing in the original Kratka approach based on a different definition of the risk coefficient $r_{\mathrm{VP}}$ measuring volatility of the portfolio. More precisely, in [14] the risk measure was defined as follows:

$$
r_{\mathrm{VP}}=R \frac{\operatorname{var}(\Delta \bar{\Pi})}{\Delta t}
$$


from which Kratka derived the following equation for the risk-adjusted option pricing methodology:

$$
\partial_{t} V+\frac{\sigma^{2}}{2} S^{2}\left(1-\mu \Gamma^{1 / 3}\right) \Gamma=r\left(V-S \partial_{S} V\right)
$$

However, this equation is not scale invariant with respect to the scaling $V \leftrightarrow \kappa V, S \leftrightarrow \kappa S$.

\section{Analysis of the risk-adjusted Black-Scholes equation}

The idea how to analyze and solve (2.19) is based on a transformation method. As it is usual in the classical Black-Scholes theory (cf. $[10,15]$ ), we consider the change of independent variables

$$
x:=\ln \left(\frac{S}{E}\right), \quad x \in \mathbb{R}, \quad \tau:=T-t, \quad \tau \in(0, T) .
$$

As (2.19) contains the term $S \Gamma=S \partial_{S}^{2} V$, it is convenient to introduce the following transformation:

$$
H(x, \tau):=S \Gamma=S \partial_{S}^{2} V(S, t)
$$

Since we have assumed that $V=V(S, t)$ is a solution to the classical Black-Scholes equation (2.21) for $\tau \in\left(0, T-t_{*}\right)$, we obtain from (2.29)

$$
H(x, \tau)=\frac{N^{\prime}\left(d_{1}\right)}{\sigma \sqrt{\tau}}, \quad d_{1}=\frac{x+\left(r+\sigma^{2} / 2\right) \tau}{\sigma \sqrt{\tau}},
$$

where $0<\tau<T-t_{*}, x \in \mathbb{R}$.

3.1. Valuation formula for option prices. Suppose for a moment that the function $H=$ $S \Gamma$ is already known. Then (2.19) can be integrated. It is an easy calculus to verify that the option price $V=V(S, t)$ is given by the formula

$$
\begin{aligned}
V(S, T-\tau)= & e^{-r\left(\tau-\tau_{*}\right)} V\left(S e^{r\left(\tau-\tau_{*}\right)}, T-\tau_{*}\right) \\
& +S \int_{\tau_{*}}^{\tau} \beta\left(H\left(\ln \left(\frac{S}{E}\right)+r(\tau-\theta), \theta\right)\right) d \theta
\end{aligned}
$$

for any $S>0$ and $\tau \in\left(\tau_{*}, T\right)$ where $\tau_{*}=T-t_{*}$. Recall that the option price $V(S, T-$ $\tau)$ for $\tau \in\left(0, \tau_{*}\right)$ can be valuated by an explicit formula for both Call and Put options, respectively (cf. $[10,15])$. More precisely, the valuation formulae for pricing European Call and Put options read as follows:

$$
\begin{aligned}
& V_{\mathrm{ec}}(S, T-\tau)=S N\left(d_{1}\right)-E e^{-r \tau} N\left(d_{2}\right), \\
& V_{\mathrm{ep}}(S, T-\tau)=E e^{-r \tau} N\left(-d_{2}\right)-S N\left(-d_{1}\right),
\end{aligned}
$$

where $d_{1}=\left(\ln (S / E)+\left(r+\sigma^{2} / 2\right) \tau\right) /(\sigma \sqrt{\tau}), d_{2}=d_{1}-\sigma \sqrt{\tau}$. 
3.2. $\Gamma$ equation. Next we derive an equation for the function $H$ on the time interval $\left(\tau_{*}, T\right)$. It turns out that the function $H(x, \tau)$ is a solution to a nonlinear parabolic equation subject to the initial and boundary conditions. More precisely, by taking the second derivative of (2.19) with respect to $x$, we obtain, after some calculations, that $H=H(x, \tau)$ is a solution to the quasilinear parabolic equation

$$
\partial_{\tau} H=\partial_{x}^{2} \beta(H)+\partial_{x} \beta(H)+r \partial_{x} H
$$

$\tau \in\left(\tau_{*}, T\right), x \in \mathbb{R}$. Henceforth, we will refer to (3.6) as a $\Gamma$ equation. A solution $H$ to (3.6) is subjected to the initial condition at $\tau=\tau_{*}$ :

$$
H\left(x, \tau_{*}\right)=\bar{H}(x), \quad x \in \mathbb{R},
$$

where $\tau_{*}=T-t_{*}$ and $\bar{H}(x)=N^{\prime}(d) /\left(\sigma \sqrt{\tau_{*}}\right)$ (see (3.3)). In the case of Call or Put options, the function $H$ is subjected to boundary conditions at $x= \pm \infty$,

$$
H(-\infty, \tau)=H(\infty, \tau)=0, \quad \tau \in(0, T) .
$$

Next we show useful bounds for a solution $H$ to the $\Gamma$ equation (3.6). Notice that any constant function $H(x, \tau) \equiv$ const is a solution to (3.6). Since $0<\max _{x \in \mathbb{R}} \bar{H}(x)<\kappa=$ $(3 /(4 \mu))^{3}$ it follows from the classical maximum principle for parabolic equations (see, e.g., [19]) that a solution $H(x, \tau)$ to the initial-boundary problem (3.6)-(3.8) satisfies the estimate

$$
0<H(x, \tau)<\kappa=(3 /(4 \mu))^{3}, \quad \text { for any } x \in \mathbb{R}, \tau \in\left(\tau_{*}, T\right) \text {. }
$$

The above estimate enables us to conclude that a solution $V(S, t)$ to the risk-adjusted Black-Scholes equation (see Definition 2.3) is indeed a solution to (2.19) on the time interval $t \in\left(0, T-\tau_{*}\right)$.

Remark 3.1. Since $0<\lambda_{-} \leq \beta^{\prime}(H) \leq \lambda_{+}$for every $H<\kappa$ where $\lambda_{ \pm}>0$ are fixed constants, the local in time existence of a classical (smooth) solution $H(x, \tau)$ to the Cauchy problem (3.6)-(3.8) is a consequence of the general theory of quasilinear parabolic equations due to Ladyzhenskaya [16]. Notice that local existence of a weak solution to (3.6)-(3.8) with an $L^{2}(\mathbb{R})$ integrable initial condition can be shown by means of Rothe's method which has been intensively studied by Kacur [13]. Global in time existence of either classical or weak solutions follows from á-priori energy bounds obtained by multiplying (3.6) with the term $\beta(H)$ and integrating over the domain $x \in \mathbb{R}$.

Remark 3.2 (free boundary problem and American-type options). Throughout the paper we assume that Call or Put options are of the European type and the underlying asset does not pay any dividend. It means that the option can be exercised only at the expiry $T$. On the other hand, American type of options are much more common in quotes markets. In this case one has to consider the effect of the free boundary (or optimal stopping time) on the valuation of option prices (see, e.g., $[15,21,22,24])$. Nevertheless, one can follow derivation of the RAPM model in order to derive a free boundary problem for valuing American type of options. A position of the free boundary can be determined by 
several methods including in particular the reduction of the problem to a solution of a certain nonlinear singular integral equation (cf. $[15,21,22])$. For the sake of simplicity, we however restrict ourselves to the study of European type of options only.

\section{Numerical scheme for full space-time discretization}

In this section we describe a full space-time discretization scheme for solving (3.6) and (3.4). The idea of construction of a numerical approximation to (3.6) is based on the finite-volume method (see, e.g., [7]).

4.1. Discretization of the $\Gamma$ equation. In order to find a numerical solution to (3.6), we have to restrict ourselves to a finite spatial interval $x \in(-L, L)$ where $L>0$ is sufficiently large. Since $S=E e^{x}$, we have restricted the interval of asset values to $S \in\left(E e^{-L}, E e^{L}\right)$. From a practical point of view, it is therefore sufficient to take $L \approx 1.5$ in order to include important values of $S$. Subsequently, we have also to modify boundary conditions (3.8). Instead of (3.8), we will consider Dirichlet boundary conditions at $x= \pm L$, that is,

$$
H(-L, \tau)=H(L, \tau)=0, \quad \tau \in\left(\tau_{*}, T\right)
$$

We take a uniform division of the time interval $[0, T]$ with a time step $k=T / m$ and a uniform division $x_{i}=i h, i=-n, \ldots, n$, of the interval $[-L, L]$ with a step $h=L / n$. To construct numerical approximation of a solution $H$ to (3.6), we derive a system of difference equations corresponding to (3.6) to be solved at every discrete time step. Difference equations involve discrete values of $H_{i}^{j} \approx H(i h, j k)$ where $j=p, \ldots, m$. Here the index $p$ corresponds to the initial time $\tau_{*}$, that is, $\tau_{*} \approx p k$. We choose the time step $k$ less than $\Delta t_{\text {opt }}($ see $(2.14))$.

Our numerical algorithm is semi-implicit in time. It means that all nonlinear terms in equations are treated from the previous time step whereas linear terms are solved at the current time level. In order to guarantee stability of the scheme, we assume the CLF condition for the time step $k$ and spatial step $h:\left(k / h^{2}\right) \lambda_{+}<1 / 2$. Such a discretization leads to a solution of linear systems of equations at every discrete time level. Now, by replacing the time derivative by the time difference, approximating $H$ in nodal points by the average value of neighboring segments, collecting all linear terms at the new time level $j$, and taking all the remaining terms from the previous time level $j-1$, we obtain a tridiagonal system subject to homogeneous Dirichlet boundary conditions imposed on new discrete values of $H^{j}$ :

$$
a_{i}^{j} H_{i-1}^{j}+b_{i}^{j} H_{i}^{j}+c_{i}^{j} H_{i+1}^{j}=d_{i}^{j}, \quad H_{-n}^{j}=0, H_{n}^{j}=0,
$$

for $i=-n+1, \ldots, n-1$, and $j=p+1, \ldots, m$, where $H_{i}^{p}=\bar{H}\left(x_{i}\right)$ and

$$
\begin{gathered}
a_{i}^{j}=-\frac{k}{h^{2}} \beta^{\prime}\left(H_{i-1}^{j-1}\right)+\frac{k}{h} r, \quad b_{i}^{j}=1-\left(a_{i}^{j}+c_{i}^{j}\right), \\
c_{i}^{j}=-\frac{k}{h^{2}} \beta^{\prime}\left(H_{i}^{j-1}\right), \quad d_{i}^{j}=H_{i}^{j-1}+\frac{k}{h}\left(\beta\left(H_{i}^{j-1}\right)-\beta\left(H_{i-1}^{j-1}\right)\right) .
\end{gathered}
$$


Since tridiagonal systems admit a simple LU-matrix decomposition, we can solve the above tridiagonal system in every time step in a fast and effective way.

4.2. Computation of option prices. Equation (3.4) is a simple updating formula once a numerical approximation of a solution $H(x, \tau)$ to the $\Gamma$ equation is known. We can use a simple trapezoidal rule for numerical integration of (3.4), that is,

$$
\begin{aligned}
V(S, T-j k)= & V\left(S e^{r(j-p) k}, T-p k\right) e^{-r(j-p) k} \\
& +S k \sum_{l=p+1}^{j} \beta(H(\ln (S / E)+r(j-l) k, l k))
\end{aligned}
$$

for $j=p+1, \ldots, m$, where $\tau_{*} \approx p k$. The value of a function $H$ at a spatial point $x=$ $\ln (S / E)+r(j-l) k \in\left[x_{i}, x_{i+1}\right]$ is computed by a piecewise linear approximation of $H$ using the neighboring values $H_{i}^{l}, H_{i+1}^{l}$.

\section{Computational results}

The purpose of this section is to discuss the application of the RAPM model to the real market option price data. We introduce a concept of the so-called implied RAPM volatility $\sigma_{\text {RAPM }}$ and the implied risk premium coefficient $R$. Furthermore, we discuss the volatility smile phenomenon and its explanation within the frame of the RAPM model.

5.1. Explanation of the volatility smile by the RAPM model. One of the most striking phenomena in the Black-Scholes theory is the so-called volatility smile phenomenon. Notice that the derivation of the classical Black-Scholes equation (2.21) relies on the assumption of a constant value of the volatility $\sigma$. On the other hand, as it might be documented by many examples observed in market options datasets (see, e.g., $[2,6,11,23]$ ), such an assumption is often violated. More precisely, the implied volatility $\sigma_{\text {impl }}$ is no longer constant and it can depend on the asset price $S$, the strike price $E$, as well as the time $t$.

In the RAPM approach we are able to explain the volatility smile analytically. The risk-adjusted Black-Scholes equation (2.19) can be viewed as an equation with a variable volatility coefficient, that is,

$$
\partial_{t} V+\frac{\bar{\sigma}^{2}(S, t)}{2} S^{2} \Gamma=r\left(V-S \partial_{S} V\right)
$$

where $\Gamma=\partial_{S}^{2} V$ and the volatility $\bar{\sigma}^{2}(S, t)$ depends itself on a solution $V=V(S, t)$ as follows:

$$
\bar{\sigma}^{2}(S, t)=\sigma^{2}\left(1-\mu(S \Gamma)^{1 / 3}\right) .
$$




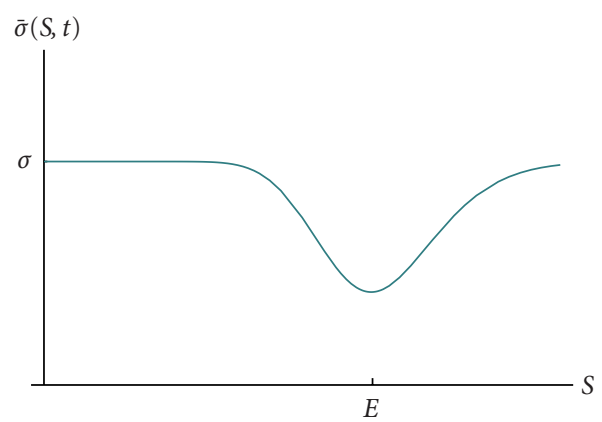

(a)

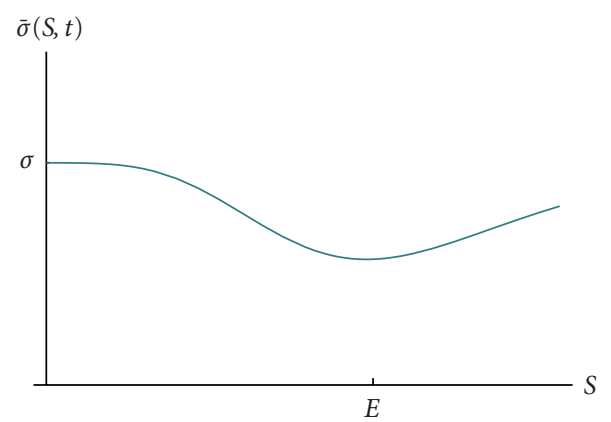

(b)

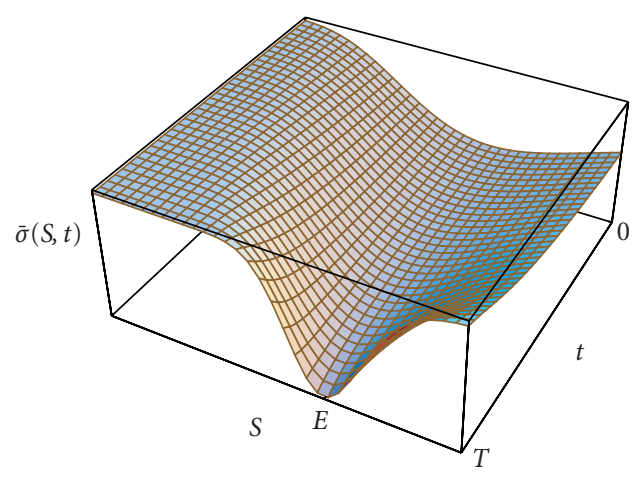

(c)

Figure 5.1. Explanation of the volatility smile. Dependence of $\bar{\sigma}(S, t)$ on $S$ is depicted in (a) for $t$ close to $T$ and in (b), for a time $0<t \ll T$. The mapping $(S, t) \mapsto \bar{\sigma}(S, t)$ is shown in (c).

In Figure 5.1 we show the dependence of the function $\bar{\sigma}(S, t)$ on the asset price $S$ and time $t$. It should be obvious that the function $S \mapsto \bar{\sigma}(S, t)$ has a convex shape near the exercise price $E$. We have used the RAPM model in order to compute values of $\Gamma=\partial_{S}^{2} V$. We chose $\mu=0.2, \sigma=0.3, r=0.011$, and $T=0.5$.

With regard to scale invariance property of the RAPM model (see Section 2.7) if we express both the asset price $S$ as well as the option price $V$ in terms of units of $E$ (i.e., we introduce scaling $s \leftrightarrow S / E$ and $v \leftrightarrow V / E$ ), then the volatility $\bar{\sigma}$ defined as in (5.2) is a function of the ratio $s=S / E$ and time $t$ only.

5.2. Modeling bid-ask spreads of option values. In real market quotes datasets there are listed two different option prices $V_{\text {bid }}<V_{\text {ask }}$ called bid and ask price representing thus offers for buying and selling options, respectively (cf. [18]). We note that in the RAPM model derived in Section 2, asset transaction costs as well as risk from an unprotected portfolio were on the side of a holder of an option, because a holder has to keep a fixed amount of options and to adjust portfolio by buying or selling assets. Having assumed such a long option position, the solution to the RAPM model (2.19) corresponds to the 


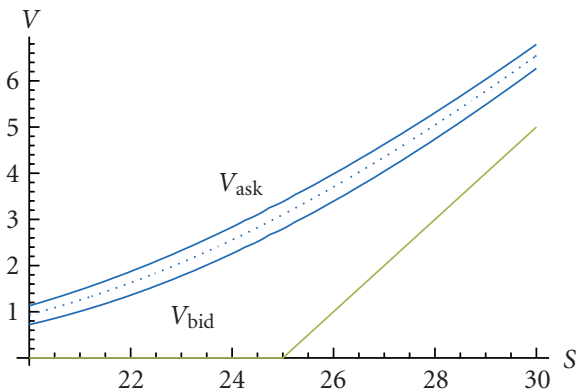

(a)

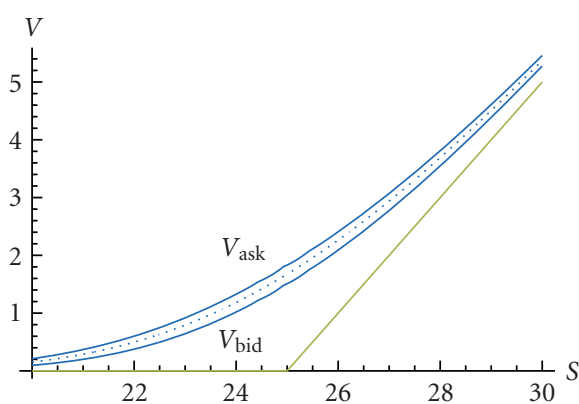

(b)

Figure 5.2. A comparison of bid and ask option prices computed by means of the RAPM model. The dotted line in the middle is the option price computed from the Black-Scholes equation. We chose (a) $\sigma=0.3, \mu=0.2, r=0.011, E=25$, and $T=1$ and (b) $T=0.3$.

bid option price $V_{\text {bid. }}$. Switching to a short positioned option we transfer both transaction costs and risk from an unprotected portfolio to the other side of an option contract. In this case, we changed the governing equation slightly—the coefficient $\mu$ has the reversed sign. It means that the RAPM equation modeling higher ask option prices reads as follows:

$$
\partial_{t} V+\frac{\sigma^{2}}{2} S^{2}\left(1+\mu(S \Gamma)^{1 / 3}\right) \Gamma=r\left(V-S \partial_{S} V\right) .
$$

The above PDE can be numerically computed exactly in the same way as the RAPM equation (2.19) for the bid option price. In fact, we only change the sign of the coefficient $\mu$ in our numerical scheme. The switching time remains the same: $t_{*}=T-C /\left(R \sigma^{2}\right)$ for valuing both $V_{\text {bid }}$ as well as $V_{\text {ask }}$ option prices.

We denote $V\left(S, t ; \sigma, t_{*}, \mu\right)$ the value of a solution to (2.19). In order to calibrate the RAPM model, we seek a pair $\left(\sigma_{\mathrm{RAPM}}, R\right)$ such that $V_{\text {bid }}=V\left(S, t ; \sigma, t_{*}, \mu\right)$ and $V_{\text {ask }}=$ $V\left(S, t ; \sigma, t_{*},-\mu\right)$, where $V_{\text {bid }}$ and $V_{\text {ask }}$ are market bid and ask option prices. It leads us to the following definition of the implied RAPM volatility and risk premium coefficients.

Definition 5.1. Let $V_{\text {bid }}, V_{\text {ask }}$ denote the market option data for the bid and ask option prices. By the implied RAPM volatility $\sigma_{\text {RAPM }}$ and implied RAPM risk premium coefficient $R$ we mean the unique values of $\sigma>0$ and $R>0$, such that $V_{\text {bid }}=V\left(S, t ; \sigma, t_{*}, \mu\right)$ and $V_{\text {ask }}=V\left(S, t ; \sigma, t_{*},-\mu\right)$, where $\mu=3\left(C^{2} R /(2 \pi)\right)^{1 / 3}, t_{*}=T-C /\left(R \sigma^{2}\right)$, and $C>0$ is transaction cost rate.

In Figure 5.2 we show a comparison of $V_{\text {bid }}$ and $V_{\text {ask }}$ option prices to the Call option payoff diagram. We also show the solution to the classical Black-Scholes equation (2.21) lying on between $V_{\text {bid }}$ and $V_{\text {ask }}$ prices. Notice that a solution $\sigma$ to the equation $V_{\text {mid }}=$ $V\left(S, t ; \sigma, t_{*}, 0\right)$, where $V_{\text {mid }}=\left(V_{\text {bid }}+V_{\text {ask }}\right) / 2$ is just the usual implied volatility $\sigma_{\text {impl }}$ (cf. $[15])$. 
Remark 5.2. In modeling bid-ask spreads, we have unambiguously associated a long positioned option with a lower bid price, and a short positioned option with a higher ask price. In a real market, it need not be so easy to switch costs and the risk to the other side of the contract. A consistent way how to calibrate the RAPM model should be to work with either one of $V_{\text {bid }}$ or $V_{\text {ask }}$ and stick to it. It turned out from the calibration of implied pairs $\left(\sigma_{\mathrm{RAPM}}, R\right)$ that $\sigma_{\mathrm{RAPM}}$ is very close to the Black-Scholes implied volatility $\sigma_{\text {impl }}$, their relative difference being less than $5.10^{-3}$ (see Section 5.3 ). Of course they need not coincide as the governing parabolic equation is nonlinear and so $(1 / 2)\left(V\left(S, t ; \sigma, t_{*},-\mu\right)+V\left(S, t ; \sigma, t_{*}, \mu\right)\right) \neq V\left(S, t ; \sigma, t_{*}, 0\right)$, in general. Nevertheless, from practical point of view, we may take $\sigma_{\mathrm{RAPM}} \approx \sigma_{\text {impl }}$ leading to calibration of the remaining parameter $\mu$ (and subsequently $R$ ) from the single equation $V_{\text {bid }}=V\left(S, t ; \sigma, t_{*}, \mu\right.$ ) only.

In order to find a pair $\left(\sigma_{\mathrm{RAPM}}, R\right)$ of the implied volatility and risk premium $R$, we have to solve the following system of nonlinear equations:

$$
F(\sigma, \mu)=\left(V_{\text {bid }}, V_{\text {ask }}\right)
$$

where the mapping $F: \mathbb{R}^{2} \rightarrow \mathbb{R}^{2}$ is defined as $F(\sigma, R)=\left(V\left(S, t ; \sigma, t_{*}, \mu\right), V\left(S, t ; \sigma, t_{*},-\mu\right)\right)$. To find a solution to (5.4), we make use of the iterative Newton-Kantorovich method (cf. $[1])$,

$$
y^{n+1}=y^{n}-\left[F^{\prime}\left(y^{n}\right)\right]^{-1} F\left(y^{n}\right), \quad n=0,1, \ldots,
$$

where $y^{n}=\left(\sigma^{n}, R^{n}\right)$ and $F^{\prime}$ is the derivative of $F$. Taking a good initial approximation $\left(\sigma^{0}, R^{0}\right)$ of an implied pair, the Newton-Kantorovich sequence $y^{n}=\left(\sigma^{n}, R^{n}\right)$ defined as in (5.5) converges to a solution $(\sigma, R)$ of $(5.4)$. In practice, we replace partial derivatives in the Jacobi matrix $F^{\prime}$ by their central difference approximations. Notice that the overall complexity of a single Newton-Kantorovich step is therefore 10 times the complexity of computation of a particular RAPM option price $V\left(S, t ; \sigma, t_{*}, \mu\right)$. In our experiments to follow, we needed (in average) 5-15 steps in the Newton-Kantorovich scheme in order to find a solution to (5.4) with accuracy less than $0.1 \%$ of the option price.

5.3. Examples of calibration of the RAPM model. In this section, we summarize results of several numerical experiments and comparison of results to market option datasets. We focus on calibration of the RAPM model. The main goal is to analyze time series of option prices and to compute the implied RAPM volatility $\sigma_{\text {RAPM }}$ and risk premium coefficient $R$. The analyzed datasets consisted of several hundreds of option prices for different exercise prices $E$ and exercise times $T$. As it was already mentioned in the previous section, computation of implied volatilities and risk premiums is rather complex because we have to solve approximately 100 PDEs per one option price record. The overall computational time ranged from 2 hours up to 6 hours of $3 \mathrm{GHz}$ Pentium CPU depending on the size of the analyzed dataset. 
As an example we considered sample datasets for Microsoft Corporation. In all studied cases, we computed the implied RAPM volatilities and risk premium coefficients. We considered a flat interest rate $r=0.02$ and a constant transaction cost coefficient $C=$ 0.01 . We also compared implied RAPM volatilities to standard implied volatilities $\sigma_{\text {impl }}$ computed by means of the classical Black-Scholes equation (2.21). It turned out that time series of $\sigma_{\text {RAPM }}$ and $\sigma_{\text {impl }}$ are almost perfectly correlated with correlation higher than 0.99 . On the other hand, in all studied cases we have $\sigma_{\mathrm{RAPM}}>\sigma_{\text {impl }}$ with the relative difference $\left(\sigma_{\text {RAPM }}-\sigma_{\text {impl }}\right) / \sigma_{\text {RAPM }}$ less than 0.005 . Notice that we have considered only Call option price records in which $V_{\text {bid }}>S_{\text {bid }}-E$.

In Figures 5.3a and 5.3b, we present the behavior of the mid value asset price $S=$ $\left(S_{\text {bid }}+S_{\text {ask }}\right) / 2$ during April 4, 2003. We chose three Call options with the same expiration date $T=$ May 17, 2003, and different expiration prices $E=23, E=25, E=30$ Figure 5.3c. The behavior of the implied volatility $\sigma_{\text {RAPM }}$ and implied risk premium $R$ is depicted Figures 5.3c and 5.3d. For Call options with expiration prices $E=25$ and $E=30$, implied risk coefficients are almost constant during the day except for the initial shock for the option with $E=30$. On the other hand, both implied risk coefficient as well as implied $\sigma_{\text {RAPM }}$ volatility are highly volatile during this day. The lowest risk (measured by $R$ ) is achieved by holding the Call option on $E=25$. These results could indicate that holding $E=25$ Call option is less risky compared to other analyzed call options.

In Figure 5.4b, we present analogous results for Microsoft stocks and Call options having a longer expiration date $T=$ January 22, 2005. Again, we chose three Call options with different expiration prices $E=20, E=25$, and $E=30$. The behavior of the implied volatility $\sigma_{\text {RAPM }}$ and implied risk premium $R$ is depicted Figures $5.4 \mathrm{c}$ and $5.4 \mathrm{~d}$. Similarly as in Figure 5.3, Call options with expiration prices $E=25$ and $E=30$ have almost constant implied risk coefficients. Interestingly enough, in the first half of the day, the implied risk coefficient $R$ for the Call option with $E=20$ is much higher compared to those corresponding to $E=25$ and $E=30$, respectively. During the second part of the day, it is jumping up and down between them. It could give some indication to an investor that the portfolio consisting of $E=25$ Call options is less risky.

Finally, in Figure 5.5 we present one-week behavior of implied volatilities and risk premium coefficients for the Microsoft Call option on $E=25$ expiring at $T=$ April 19, 2003. In the beginning of the investigated period, the risk premium coefficient $R$ was rather high and fluctuating. On the other hand, it tends to a flat value of $R \approx 5$ at the end of the week.

\section{Discussion}

We introduced a new model for pricing derivative securities in the presence of both transaction costs as well as the risk from unprotected portfolio. The option prices can be computed from a solution to a nonlinear parabolic PDE. The governing equation extends the classical Black-Scholes equation and Leland's equation to the case when the risk from unprotected portfolio is taken into account. It is a fully nonlinear parabolic equation in which a diffusion coefficient representing the volatility nonlinearly depends on the solution itself giving rise to analytically explain the volatility smile phenomenon. We have shown how this equation can be approximated by a stable numerical scheme. We 
254 The risk-adjusted pricing methodology

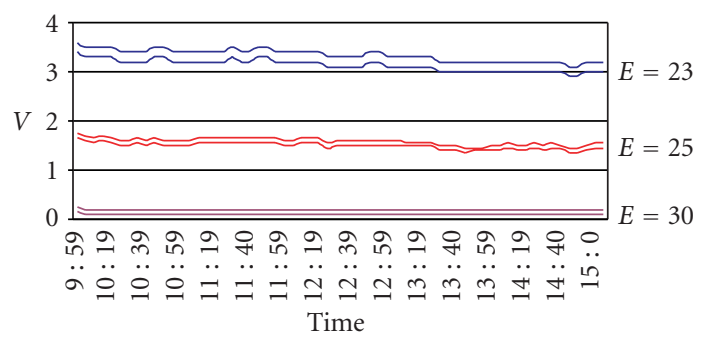

(a)

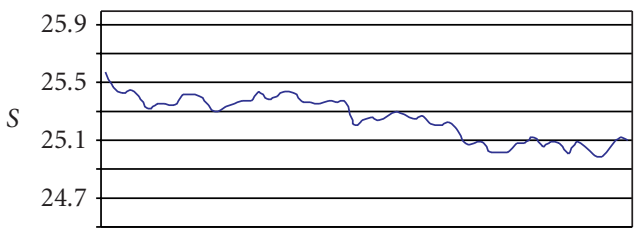

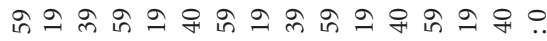

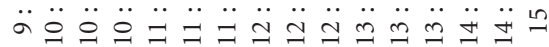

Time

(b)

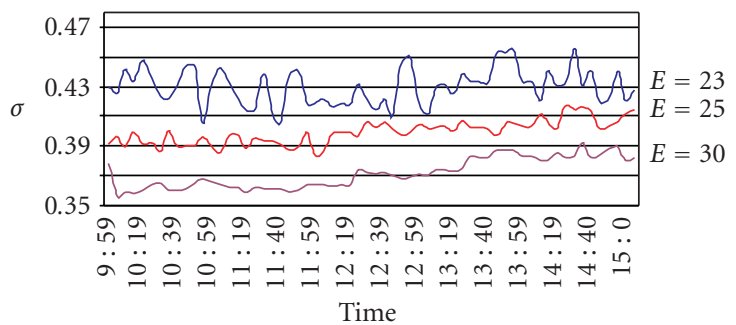

(c)

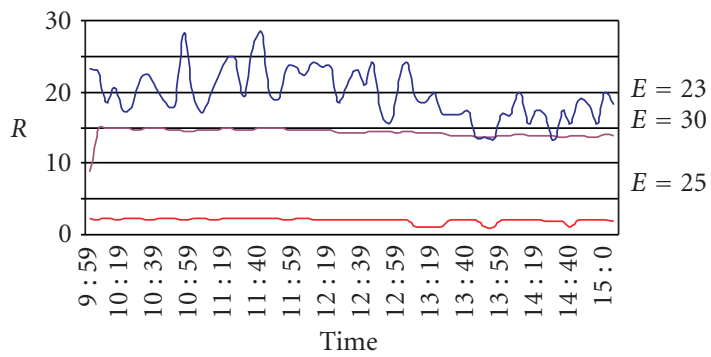

(d)

Figure 5.3. Intraday behavior of Microsoft stocks (April 4, 2003) and shortly expiring Call options with expiry date April 19, 2003, with computed implied volatilities $\sigma_{\text {RAPM }}$ and risk premium coefficients $R$. 


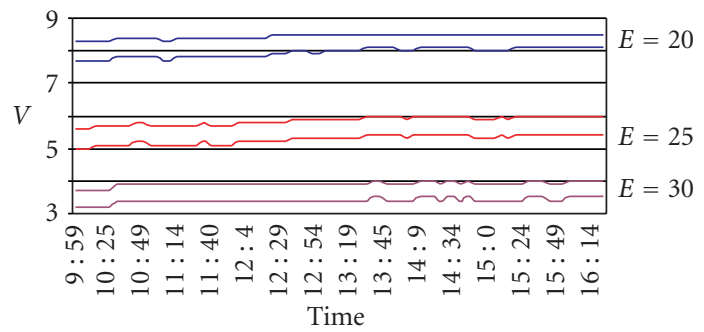

(a)

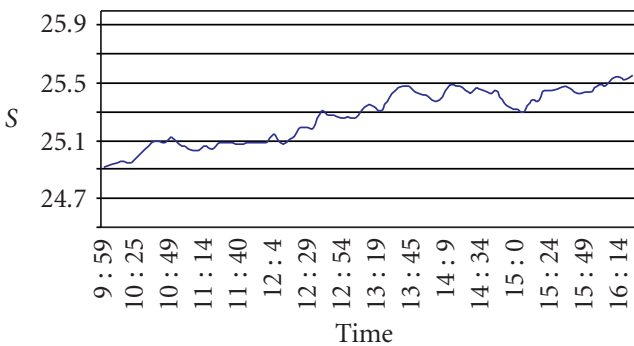

(b)

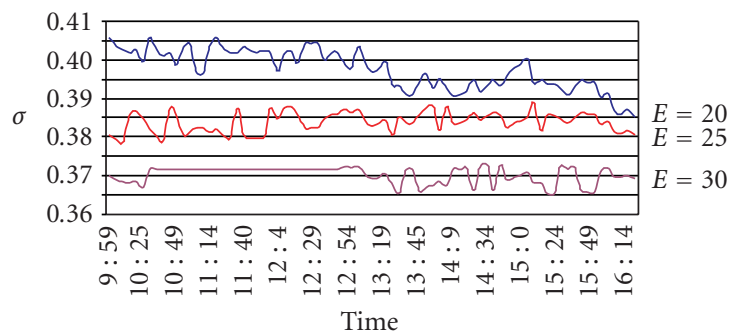

(c)

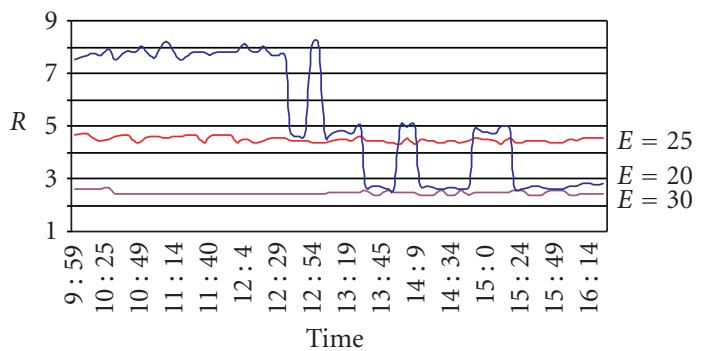

(d)

Figure 5.4. Intraday behavior of Microsoft stocks (April 17, 2003) and Call options with long expiration date January 22, 2005, with computed implied volatilities $\sigma_{\text {RAPM }}$ and risk premiums $R$. 
256 The risk-adjusted pricing methodology

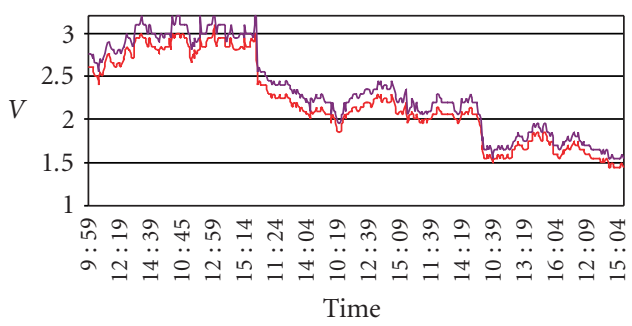

(a)

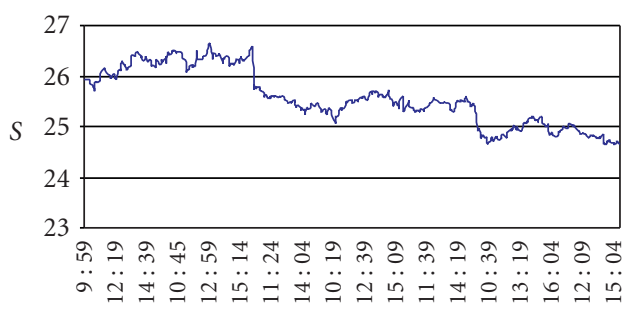

Time

(b)

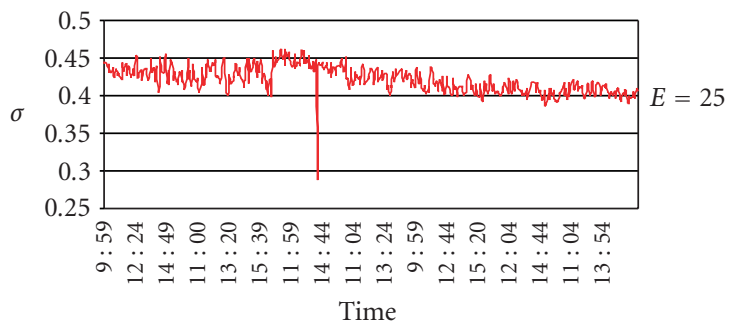

(c)

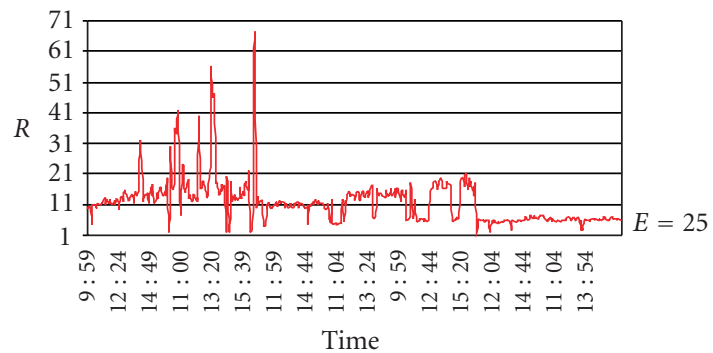

(d)

Figure 5.5. One week behavior of Microsoft stocks (March 20-27, 2003) and Call options with expiration date April 19, 2003. Computed implied volatilities $\sigma_{\text {RAPM }}$ and risk premiums $R$. 
have performed extensive numerical testing of the model and compared the results to real option market data. Furthermore, we introduced a concept of the so-called implied RAPM volatility and implied risk premium coefficients. We have computed these implied quantities for sample option datasets and we have indicated how these implied factors can be used in qualitative analysis of option market datasets.

\section{Acknowledgments}

The authors are thankful to the anonymous referee for valuable comments and suggestions. This work was supported by the VEGA Grant 1/9154/02.

\section{References}

[1] E. L. Allgower and K. Georg, Numerical Continuation Methods, Springer Series in Computational Mathematics, vol. 13, Springer-Verlag, Berlin, 1990.

[2] M. Avellaneda and A. Paras, Dynamic hedging portfolios for derivative securities in the presence of large transaction costs, Appl. Math. Finance 1 (1994), 165-193.

[3] _ Pricing and hedging derivative securities in markets with uncertain volatilities, Appl. Math. Finance 2 (1995), 73-88.

[4] M. Baxter and A. Rennie, Financial Calculus: An Introduction to Derivative Pricing, Cambridge University Press, Cambridge, 1996.

[5] F. Black and M. Scholes, The valuation of options contracts and a test of marker efficiency, J. Finance 27 (1972), 399-417.

[6] J. Duque and D. Paxson, Implied volatility and dynamic hedging, Review of Futures Markets 13 (1994), 381-421.

[7] R. Eymard, M. Gutnic, and D. Hilhorst, The finite volume method for an elliptic-parabolic equation, Acta Math. Univ. Comenian. 67 (1998), no. 1, 181-195.

[8] P. Grandits and W. Schachinger, Leland's approach to option pricing: the evolution of a discontinuity, Math. Finance 11 (2001), no. 3, 347-355.

[9] T. Hoggard, A. E. Whalley, and P. Wilmott, Hedging option portfolios in the presence of transaction costs, Adv. in Futures and Options Res. 7 (1994), 21-35.

[10] J. Hull, Options, Futures and Other Derivative Securities, Springer Series in Computational Mathematics, Prentice Hall, New York, 1989.

[11] H. Johnson and D. Shanno, Option pricing when the variance is changing, Journal of Financial and Quant. Anal. 22 (1987), 143-151.

[12] M. Kabanov and M. M. Safarian, On Leland's strategy of option pricing with transaction costs, Finance Stoch. 1 (1997), 239-250.

[13] J. Kacur, Method of Rothe in Evolution Equations, Teubner-Texte zur Mathematik, vol. 80, BSB B. G. Teubner Verlagsgesellschaft, Leipzig, 1985.

[14] M. Kratka, No mystery behind the smile, Risk 9 (1998), 67-71.

[15] Y. K. Kwok, Mathematical Models of Financial Derivatives, Springer Finance, Springer-Verlag Singapore, Singapore, 1998.

[16] O. A. Ladyzhenskaya, V. A. Solonnikov, and N. N. Ural'ceva, Linear and Quasilinear Equations of Parabolic Type, vol. 23, American Mathematical Society, Rhode Island, 1968.

[17] H. E. Leland, Option pricing and replication with transactions costs, J. Finance 40 (1985), 12831301.

[18] I. Pena, G. Rubio, and G. Serna, Smiles, bid-ask spreads and option pricing, European Financial Management, forthcoming, 2001.

[19] M. H. Protter and H. F. Weinberger, Maximum Principles in Differential Equations, SpringerVerlag, New York, 1984. 
[20] D. M. Salopek, American Put Options, Pitman Monographs and Surveys in Pure and Applied Mathematics, vol. 84, Addison Wesley Longman, Harlow, 1997.

[21] D. Ševčovič, Analysis of the free boundary for the pricing of an American call option, European J. Appl. Math. 12 (2001), no. 1, 25-37.

[22] R. Stamicar, D. Ševčovič, and J. Chadam, The early exercise boundary for the American put near expiry: numerical approximation, Canad. Appl. Math. Quart. 7 (1999), no. 4, 427-444.

[23] R. G. Tompkins, Implied volatility surfaces: uncovering regularities for options on financial futures, The European Journal of Finance 7 (2001), 198-230.

[24] P. Wilmott, J. Dewynne, and S Howison, Option Pricing: Mathematical Models and Computation, Oxford Financial Press, UK, 1995.

Martin Jandačka: Department of Economic and Financial Models, Faculty of Mathematics, Physics and Informatics, Comenius University, 84248 Bratislava, Slovak Republic

E-mail address: jandacka@fmph.uniba.sk

Daniel Ševčovič: Institute of Applied Mathematics, Faculty of Mathematics, Physics and Informatics, Comenius University, 84248 Bratislava, Slovak Republic

E-mail address: sevcovic@fmph.uniba.sk 


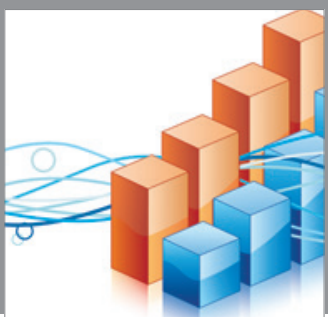

Advances in

Operations Research

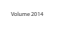

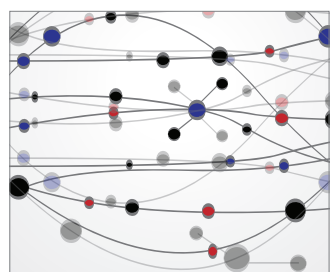

\section{The Scientific} World Journal
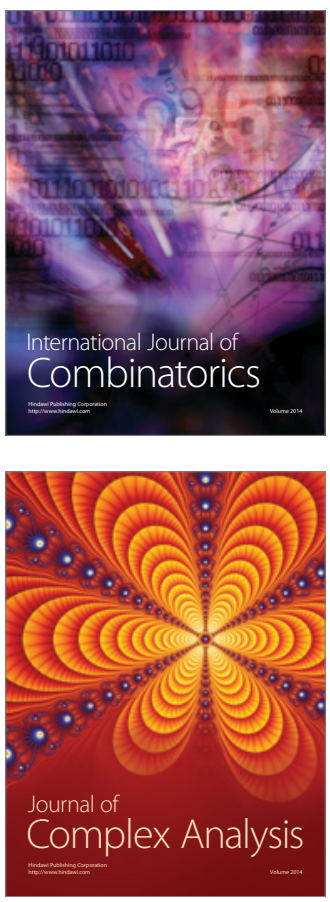

International Journal of

Mathematics and

Mathematical

Sciences
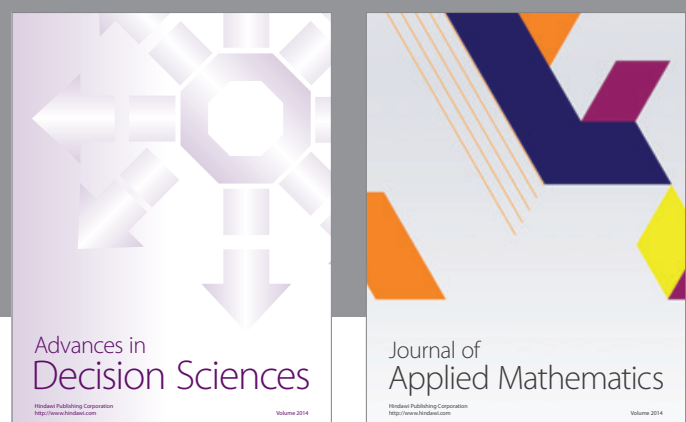

Journal of

Applied Mathematics
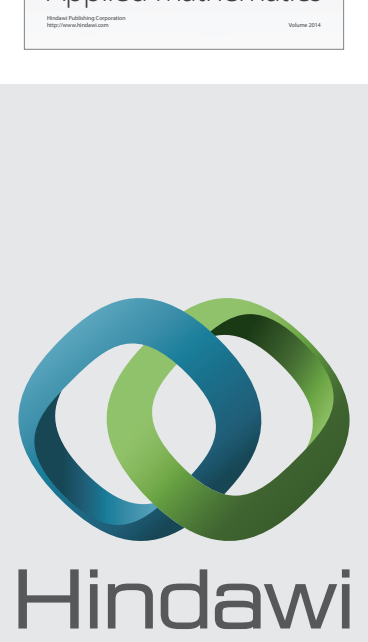

Submit your manuscripts at http://www.hindawi.com
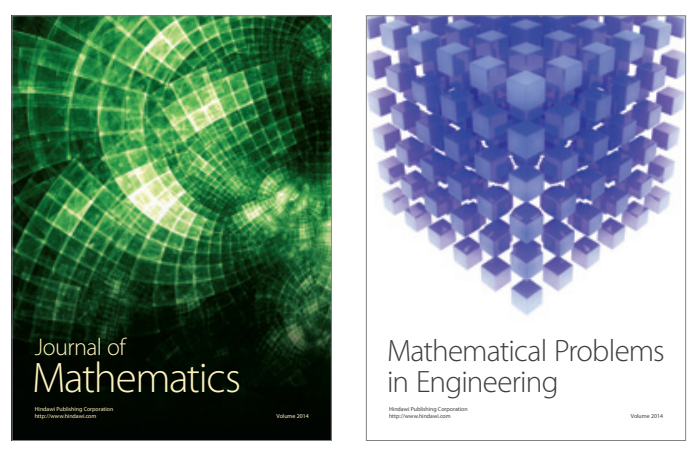

Mathematical Problems in Engineering
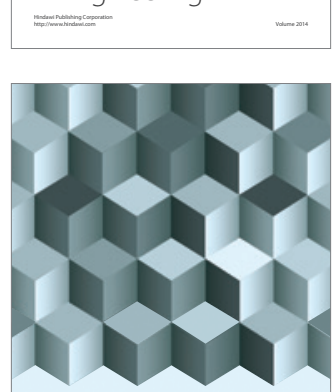

Journal of

Function Spaces
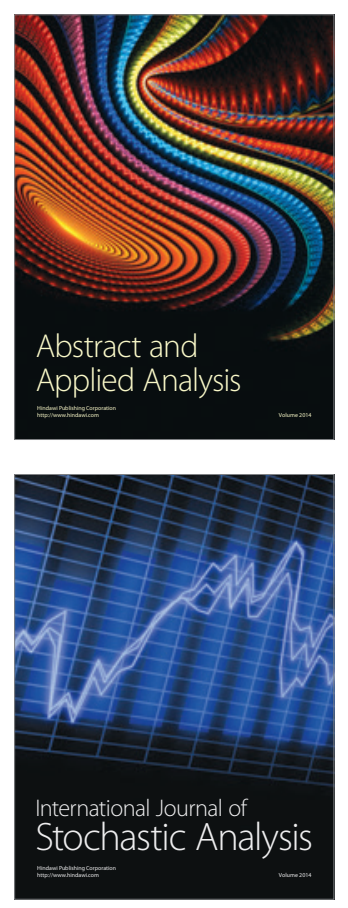

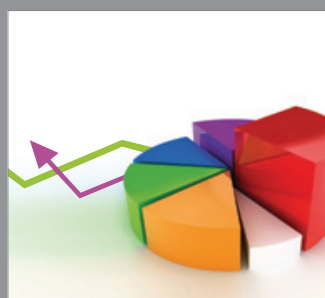

ournal of

Probability and Statistics

Promensencen
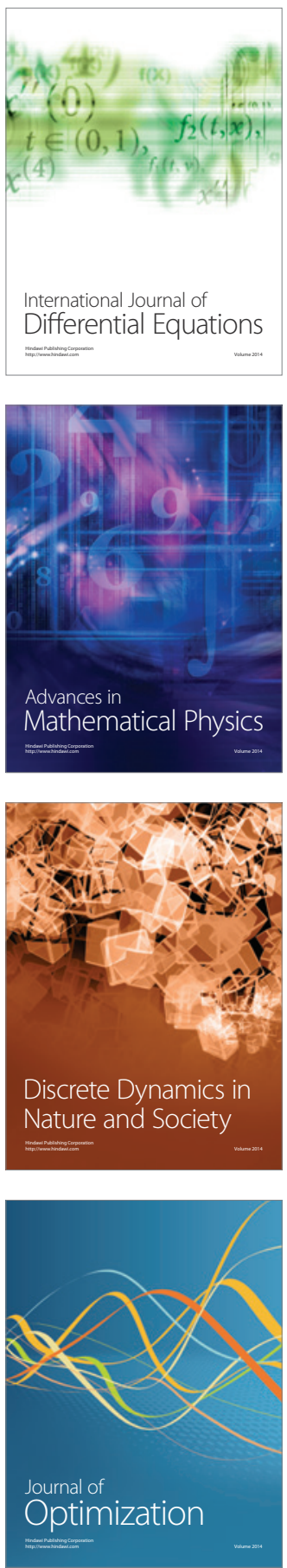